\title{
SMALL-SCALE MAGNETIC STRUCTURES AND SUPERGRANULATION FLOWS
}

\section{ZHANG YI, EBERHART JENSEN AND ODDBJøRN ENGVOLD}

Institute of Theoretical Astrophysics, University of Oslo Oslo, Norway

\begin{abstract}
Relations between horizontal flow and filigree structure obtained from high-resolution observations $\left(\sim \frac{1}{4}\right)$ are discussed. The magnetic flux elements move with the horizontal flow in the supergranulation network, and as downdrafts are approached both magnetic flux and intensity gradually decrease. Reasons for this disappearance are discussed. If the photosphere actually is a turbulent magneto-plasma, as suggested by the good coupling between the magnetic flux elements and the flow field, the integrated magnetic flux in the background field will exceed the flux in the discrete kilogauss structures.
\end{abstract}

\section{RESUMÉ OF RECENT RESULTS}

High resolution observations recently obtained with the Swedish Vacuum Solar Telescope at La Palma have been used to study interactions between small scale magnetic flux elements and the flows in low-activity photospheric regions (Yi 1992; Yi and Engvold 1992). Details on these observations and the data processing are given in the paper by $\mathrm{Yi}$ and Engvold (1992). The main conclusions from these studies are:

1. Solar filigree structure (Dunn and Zirker 1973) shows very closely a one-to-one correspondence with magnetic flux density $\alpha B_{\|}$(the factor $\alpha$ represents the area filling factor). The filigree structure is therefore suitable for mapping and tracing of magnetic flux concentrations in the solar photosphere.

2. The magnetic flux elements move with the local horizontal flow of the supergranulation network.

3. Magnetic elements are hardly ever located exactly above the rather sharply defined downdrafts at the boundaries of supergranulation cells, and a pronounced and gradual decrease in $\alpha B_{\|}$is observed as the flux elements approach the downdrafts. The decrease of $\alpha B_{\|}$, as well as the filigree intensity, with horizontal flow velocity is detectable only within about 2 arcsec from the downdrafts. One explanation for this effect may be that the field becomes increasingly more tilted upon approaching the downdraft (the Zeeman signal gives only the line-of-sight component of 
the field) (Yi and Engvold, 1992). Another possibility suggested here is that the elements fade by dissipation.

4. The mean values of the observed magnetic flux density $\left(\alpha B_{\|}\right)$, decrease linearly towards the magnetic polarity inversion in the photosphere below quiescent prominences.

\section{DISCUSSION}

Some interesting consequences of these new results are noticed. These are related to prominence structure and dynamics, the unobservable, supposedly turbulent, background magnetic fields, and the observed disappearance of magnetic flux in the solar photosphere.

\subsection{Relation to Solar Prominences}

It is known that large quiescent filaments, and their so-called footpoints, are located above, and very close to, the supergranular cell boundaries and downdrafts (Ploceniak and Rompolt 1973; Yi 1992). The convective motions of the photosphere therefore lead to a continuous restructuring of the supporting magnetic fields, which inevitably will influence strongly both the structure and dynamics of solar prominences.

The connection between the flowing photospheric plasma and the fact that the magnetic fields of solar prominences are rooted in the photosphere, means that the flow field will strongly determine the formation and evolution of prominences. In light of the characteristic pattern of magnetic flux elements moving with the photospheric flows, one may picture prominences as large "centipedes on jelly". The dynamics and lifetimes of prominence structures are inevitably a result of the dynamics of the subjacent photosphere.

\subsection{The Magnetic Properties of the Photospheric Plasma}

\subsubsection{The magnetic Reynolds number}

The observations show, within the accuracy of the measurements, that the magnetic flux elements move with the horizontal convective flows, suggesting that the magnetic fields are "frozen" to the plasma. This indicates that the magnetic Reynolds number must be large.

The conventional electrical resistivity in the photospheric plasma is to a reasonable accuracy given by;

$$
\eta=10^{13} T^{-\frac{2}{2}} \quad\left[\mathrm{~cm}^{2} s^{-1}\right]
$$

If we take the smallest resolved elements to have a characteristic scale of $\ell \approx 100 \mathrm{~km}$ (Stenflo 1989), a velocity of $V \approx 350\left[\mathrm{~m} \mathrm{~s}^{-1}\right]$, and a temperature in the observed layer equal to $5000 \mathrm{~K}$, the magnetic Reynolds number becomes;

$$
R_{m}=\frac{V \ell}{\eta}=310^{4}
$$

This value of $R_{m}$ shows that the condition for "frozen-in" fields is satisfied. 
The decay term in the induction equation is negligible compared to the convective term, $\nabla \times(v \times B)$. The corresponding decay time becomes;

$$
\tau_{\text {decay }}=\frac{R_{m} L}{V} \sim 170 \text { days }
$$

\subsubsection{Dissipation of magnetic flux elements?}

Given the decay time according to the classical picture discussed above, dissipation of magnetic flux should be negligible within the time scale of our observations.

However, in a compressible turbulent plasma the presence of waves (Langmuir-waves) will increase the resistivity by a factor of $10^{4}-10^{5}$ (Bohmresistivity, cf. Krause and Rädler (1980)). Then $R_{m}$ will be of the order of unity, and the decay-time reduces to between 5 and 50 minutes. This decaytime is comparable to the time scale of the apparent fading of flux elements near downdrafts in the present series of observations. We may therefore ask if we really witness the dissipation of magnetic flux elements when we see the filigree structures fade when getting closer to the downdrafts than some 2 arcsec?

\subsubsection{Tilting of the flux tubes?}

Another scenario for the apparent flux disappearance could be that the flux tubes become increasingly tilted relative to the radial direction, and that they actually are pulled down into the downdraft regions.

A conventional argument against this behaviour is that buoyancy forces are sufficiently strong to keep the tubes vertical even with the horizontal velocity changing with depth. However, this argument might not be valid since magnetic buoyancy may be severely reduced for flux tubes that are semitransparent (slightly cooler than the environments) or force-free (Jensen 1992).

\subsubsection{A "turbulent" background magnetic field?}

A consequence of a possible magnetic turbulent photospheric plasma is that there is equipartition between the kinetic energy density and the turbulent magnetic field. From the observed broadening of photospheric lines one finds a non-thermal velocity of $1.0-2.0\left[\mathrm{~km} \mathrm{~s}^{-1}\right]$, which is associated with regular granulation. With a mass density of $\rho \simeq 310^{-7}\left[\mathrm{~g} \mathrm{~cm}^{-3}\right]$, one derives an equipartition magnetic field of $150-300$ [gauss], for the background magnetic flux. This value would imply that a majority of the solar photospheric flux is contained in this background field.

Let there be $N$ magnetic flux elements of field strength $B_{k G} \approx 1.3 \mathrm{kG}$ and diameter $D_{k G} \sim 100 \mathrm{~km}$, per supergranular cell of diameter $D_{s g} \sim 30000 \mathrm{~km}$ and average field strength $B_{b g} \approx 150$ [gauss], and the ratio of magnetic flux contained in the background field to that of the kG-structures becomes:

$$
R_{f l u x}=\frac{\left|B_{b g}\right|}{N\left|B_{k G}\right|}\left(\frac{D_{s g}}{D_{k G}}\right)^{2}
$$


For values of $N=100$ and 1000 , one then gets $R_{f / u x}=100$ and 10 , respectively. This value exceeds by a large factor the currently assumed value $\sim 0.1$ (Stenflo 1989).

\section{POSSIBLE CONSEQUENCES OF THE PROPOSED SCENARIOS}

The lifetimes and motions of prominence structures are the result of the dynamic character of the photosphere where the magnetic footpoints are rooted. A result of the present picture of the prominence-photosphere relation is that the small-scale and medium-scale features of prominences are continuously undergoing re-organization, formation and destruction, in the course of minutes to a few hours. On this background, the validity of static approximations in modelling of prominences must be carefully examined.
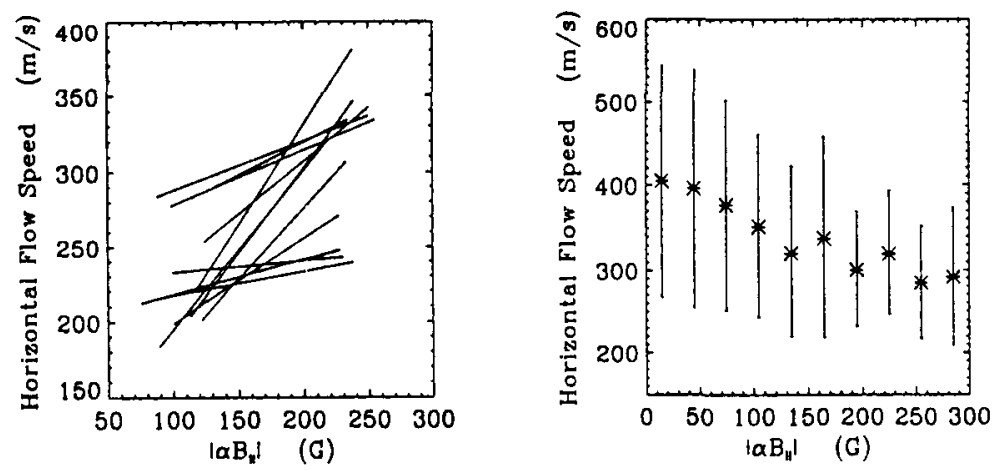

Fig. 1. (a) Local horizontal flow speed versus magnetic flux density $\alpha B_{\|}$ for a large number of magnetic structures situated within 2 arcsec from downdraft regions. (b) The same for all magnetic flux elements within the entire field of view, in agreement with the result of Title et al. 1992.

The observations have shown that magnetic elements are fading and disappearing in the downdraft regions of the supergranulation network. This phenomenon could have to do with a compressible turbulent magneto-plasma, implying the presence of Langmuir waves and turbulent dissipation. (With this picture in mind, one might speculate if the so-called high velocity events, also denoted explosive events, of the solar transition region are signatures of such dissipation of the magnetic field. These events occur predominantly along the downdraft regions of the supergranulation network (Dere et al. 1991).) An alternative mechanism for the disappearance of the flux elements is that they are being pulled down into the subphotospheric regions by the flow. Whatever the reason FOR the flux disappearance has, we are here witnessing the removal of magnetic flux from the photosphere.

\section{REFERENCES}

Dunn, R B, and Zirker, J B: 1973, Solar Phys. 33, 281. 
Dere, K P, Bartoe, J-D F, Brueckner, G E, Ewing, J, and Lund, P: 1991, Journal of Geophys. Res. 86, 9399.

Jensen E: 1992 (In preparation).

Krause F, and Rädler K-H: 1980 Mean Field Magnetohydrodynamics and Dynamo Theory Pergamon Press.

Stenflo, J O: 1989, The Astronomy and Astrophysics Review 1, 3.

Title A M, Topka K P, Tarbell T D, Schmidt W, Balke C, and Scharmer G: 1992, Astrophys. J. 393, 782.

Yi, Z: 1992 Dr.Sci.-Thesis. University of Oslo.

Yi, Z, and Engvold, O: 1992, Solar Physics (in press). 